\title{
Myroides odoratimimus, una causa infrecuente de infección de tejidos blandos y osteomielitis
}

\author{
Myroides odoratimimus, a rare cause of soft tissue infection and osteomyelitis. \\ Case report
}

Rodrigo Cruz Choappa ${ }^{1,2}$, Eduardo López 3 , Miranda Ocara ${ }^{3}$ y Yerko Leiva²

\author{
1 Unidad de Infectología. Hospital Carlos van Buren \\ ${ }^{2}$ Laboratorio Clínico, Universidad de Valparaíso. \\ ${ }^{3}$ Facultad de Medicina, Universidad de Valparaíso. \\ Los autores no tienen conflictos de intereses. \\ Financiamiento: Universidad de Valparaíso.
}

Recibido: 29 de octubre de 2020 / Aceptado: 17 de marzo de 2021

\section{Resumen}

Las infecciones por bacterias gramnegativas del género Myroides son muy poco frecuentes y generalmente afectan la piel y tejidos blandos de pacientes con algún grado de inmunocompromiso. Presentamos un caso de una mujer de 23 años, con antecedentes de mielomeningocele operado y pie bot, que cursó con una infección profunda de la extremidad inferior derecha por Myroides odoratimimus. La identificación de especie se realizó con técnica de MALDI-TOF. El tratamiento fue inicialmente con meropenem y ajustado a ciprofloxacina, junto con realizar una amputación supramaleolar derecha.

Palabras clave: Myroides spp.; osteomielitis; resistencia antimicrobiana.

\section{Introducción}

E 1 género Myroides, excluido del género Flavobacterium debido a importantes diferencias genómicas y fenotípicas ${ }^{1}$, está compuesto por dos especies clínicamente relevantes, $M$. odoratus y M. odoratimimus ${ }^{1}$. Son bacilos gramnegativos aeróbicos, no móviles, cuyas colonias pueden tener una pigmentación amarilla y un olor frutal característico ${ }^{1}$. Tradicionalmente, se le ha considerado un patógeno oportunista, encontrándose en forma ubicua en el medioambiente, especialmente en entornos acuáticos y en el suelo, sin embargo, se han aislado desde una amplia variedad de sustratos ${ }^{2}$.

Desde 1920, cuando se identificó por primera vez como Flavobac-

\section{Abstract}

Infections due to Gram-negative bacteria of the genus Myroides are very rare and generally affect the skin and soft tissues of patients with some degree of immunocompromise. We present a case of a 23 -yearold patient with a history of myelomeningocele surgically resolved at 3 years of age and bot foot, who presented with a deep infection of the right lower extremity by Myroides odoratimimus. The species identification was carried out with MALDI-TOF and the treatment was initially carried out with meropenem and finally then ciprofloxacin, in addition to right supramaleolar amputation.

Keywords: Myroides spp.; osteomyelitis; antimicrobial resistance.

terium odoratus, se han descrito alrededor de 60 casos de infecciones con rol patogénico bien documentado ${ }^{3}$. Aunque generalmente se ha descrito en infecciones de pacientes con antecedentes de diabetes mellitus, cirrosis, enfermedad pulmonar obstructiva crónica o terapia prolongada con corticosteroides, también se han documentado casos en individuos inmunocompetentes ${ }^{2,3}$. Las localizaciones reportadas son principalmente infecciones de piel y tejidos blandos, sin embargo, tambien se han descrito casos de endocarditis, ventriculitis, además de dos brotes, uno de infección del tracto urinario y otro de infección del torrente sanguíneo asociada a catéter venoso central ${ }^{4-6}$. Las especies de este género son frecuentemente resistentes a múltiples antimicrobianos, con mecanismos aún no completamente dilucidados?. 
Se presenta un caso clínico de una infección de tejidos blandos y osteomielitis de extremidad inferior por $M$. odoratimimus, en una mujer joven con antecedentes de pie bot y una úlcera plantar crónica.

\section{Caso clínico}

Mujer de 23 años, domiciliada en Valparaíso, con antecedentes de un mielomeningocele operado a los tres años de edad y varias cirugías por pie bot. Consultó en la unidad de emergencia de adultos del hospital Carlos van Buren de Valparaíso por un cuadro clínico de cinco días de evolución, caracterizado por un aumento de volumen y dolor en el pie derecho, coloración violácea en el primer ortejo y dos úlceras, una en la cara lateral y otra, de carácter crónica, en la zona plantar del mismo pie. Un día previo a la consulta se agregó fiebre de $39^{\circ} \mathrm{C}$.

En el laboratorio de ingreso destacó en el hemograma un hematocrito de $29,4 \%$, leucocitosis de 14.100 céls/ $\mathrm{mm}^{3}$ con $81 \%$ de polimorfonucleares, PCR: $284 \mathrm{mg} / \mathrm{l}$ y un lactato en sangre de $17 \mathrm{mg} / \mathrm{dl}$. Se realizó una angiotomografía de extremidades inferiores destacando una lesión ulcerativa en la región plantar derecha con penetración profunda a tejidos blandos y con signos de osteomielitis, sin alteración significativa en la vascularización de la extremidad. Evaluada por el equipo quirúrgico, y ante sospecha de una gangrena del pie derecho, fue sometida a una amputación supra-maleolar derecha. Se mantuvo desde su ingreso con terapia antimicrobiana i.v. con ceftriaxona $2 \mathrm{~g}$ al día y metronidazol $500 \mathrm{mg}$ cada $8 \mathrm{~h}$.

Al quinto día de hospitalización se realizó un aseo quirúrgico con una toma de muestra de secreción a través del muñón, cuyo cultivo mostró abundante desarrollo de Myroides spp. (identificado a través de Vitek 2 GN de bioMérieux), resistente a amikacina $(\mathrm{CIM}>64)$, gentamicina $(\mathrm{CIM}>16)$, ceftazidima $(\mathrm{CIM}>64)$ e imipenem

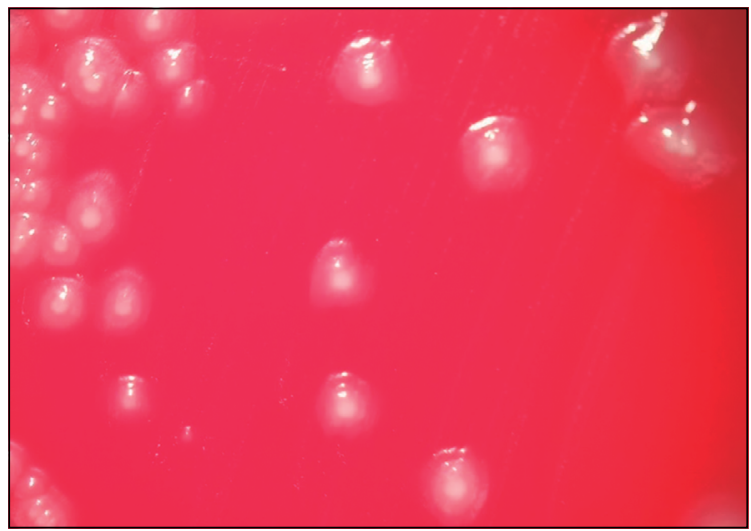

Figura 1. Colonias de Myroides odoratimimus. Se observan colonias de 3 a $4 \mathrm{~mm}$ de diámetro en agar sangre, sin hemólisis.
$(\mathrm{CIM}>16)$ y sensible a ciprofloxacina $(\mathrm{CIM}=1)$, cefepime $(\mathrm{CIM}=8)$ y meropenem $(\mathrm{CIM}=4)$. Se decidió mantener el esquema antimicrobiano ya que la paciente evolucionó clínicamente estable y afebril, sin embargo, con actividad inflamatoria elevada y secreción turbia por la herida operatoria.

Al $12^{\circ}$ día de hospitalización ingresó nuevamente a pabellón quirúrgico para aseo y toma de una muestra de tejido óseo del canal medular tibial. De dicha muestra hubo abundante desarrollo bacteriano, siendo identificado a través de Vitek 2 GN como Myroides spp. con igual patrón de resistencia al aislado anterior. El cultivo fue posteriormente identificado a través de MALDI Biotyper ${ }^{\circledR}$ (Bruker Daltonics) como M. odoratimimus. El estudio histopatológico informó una inflamación inespecífica de los tejidos y signos de osteomielitis.

Se ajustó el tratamiento antimicrobiano a meropenem i.v. $2 \mathrm{~g}$ cada $8 \mathrm{~h}$. La paciente evolucionó favorablemente, con disminución de los parámetros inflamatorios de laboratorio. Finalmente, se realizó el cierre de muñón y se dio de alta con ciprofloxacina oral $750 \mathrm{mg}$ cada $12 \mathrm{~h}$, recibiendo en total seis semanas de tratamiento antimicrobiano.

\section{Diagnóstico microbiológico}

La muestra ósea se cultivó durante $24 \mathrm{~h}$ a $37^{\circ} \mathrm{C}$ en caldo tioglicolato. Posteriormente, se realizó la siembra del caldo en agar sangre, MacConkey y chocolate, obteniéndose un cultivo puro de colonias de bacilos gramnegativos no fermentadores de tamaño mediano (Figura 1), levemente pigmentados, no hemolíticas, oxidasa, catalasa y ureasa positiva, y con un característico olor frutal. La identificación a nivel de género se llevó a cabo utilizando las tarjetas para bacterias gramnegativas y el sistema semi-automatizado para identificación de microorganismos Vitek 2 GN Compact (BioMérieux). Se obtuvo la identificación de Myroides sp. con un $98 \%$ de precisión. Posteriormente la especie fue identificada a través de espectrometría de masas MALDI Biotyper ${ }^{\circledR}$ (Bruker Daltonics) como M. odoratimimus.

El estudio de susceptibilidad antimicrobiana se realizó por microdilución automatizada con Vitek 2 Compact (BioMérieux), con tarjeta AST-N249, corroborándose según documento CLSI M100 Ed30. Tabla 2B-5.

\section{Discusión}

Las infecciones por el género Myroides son poco frecuentes, reportándose hasta la fecha 60 casos; sin embargo, 29 de ellos han ocurrido durante la última década $^{3}$ considerándose una infección emergente en la actualidad ${ }^{3,8}$. La mayoría de los casos corresponden a afecciones de piel y tejidos blandos, tal como en el 
caso de nuestra paciente, que cursó con un compromiso cutáneo profundo y posteriormente con una osteomielitis, cuya confirmación se efectuó por el cultivo de tejido, el que coincidió con los de secreción de la herida, ambos tomados posterior a la cirugía de amputación ${ }^{3,8,9}$.

Además de $M$. odoratus y $M$. odoratimimus (especies más comunes), se han identificado varias otras, como $M$. pelagicus, $M$. profundi y $M$. marinus, que han sido aisladas desde agua de mar, aunque no se han documentado como agentes de infecciones en humanos ${ }^{3,9}$. Sí se han reportado infecciones por $M$. injenensis y M. phaeus ${ }^{3,10}$. El caso aquí presentado, según nuestro conocimiento, es el primero reportado en Chile.

Si bien, este agente afecta principalmente a pacientes con algún grado de inmunocompromiso ${ }^{3,9}$, nuestra paciente presentaba como factor de riesgo una úlcera plantar de larga evolución, eventual puerta de entrada para la infección ósea y de tejidos blandos. Probablemente, el insuficiente aseo de la herida crónica en el pie afectado, dada la dificultad para movilizarse y su condición social, facilitó la infección. En personas previamente sanas este agente ha sido descrito sólo en el caso de un niño con una herida provocada por la mordedura de un cerdo ${ }^{8}$.

El diagnóstico de especie en este género debe rea- lizarse necesariamente por espectrometría de masas MALDI-TOF o secuenciación del gen ARN 16 $3 \mathrm{~S}^{3,7,10}$. En nuestra paciente, el género se identificó por el método automatizado Vitek 2 y la especie, por espectrometría de masas MALDI-TOF.

El tratamiento de la osteomielitis requiere frecuentemente un manejo quirúrgico, y el antibacteriano elegido debe ser ajustado a la susceptibilidad informada por el laboratorio $^{3,9,11}$. La mayoría de las cepas de Myroides spp. son resistentes a los $\beta$-lactámicos, incluidos aztreonam, carbapenémicos y aminoglucósidos; sin embargo, suelen ser sensibles a quinolonas y cotrimoxazol ${ }^{3,9,11}$. La resistencia antimicrobiana se debe a más de un mecanismo, incluyendo betalactamasas, bombas de expulsión de fármacos, la formación de biopelícula y disminución de porinas, este último relacionado con la resistencia a imipenem $^{11,12}$. En el caso presentado, la cepa era sensible a meropenem y ciprofloxacina, razón por la cual fue tratada con estos antibacterianos, con buena respuesta clínica y de laboratorio. Se utilizaron dosis altas de meropenem considerando la gravedad de la infección, para aumentar la concentración en el tejido óseo y dada la escasa evidencia publicada sobre la dosificación de antibacterianos en estos agentes.

\section{Referencias bibliográficas}

1.- Vancanneyt M, Segers P, Torck U, Hoste B, Bernardet J F, Vandamme P. Reclassification of Flavobacterium odoratum (Stutzer 1929) strains to a new genus, Myroides, as Myroides odoratus comb. nov. and Myroides odoratimimus sp. nov. Int J Syst Bacteriol 1996; 46: 926-32. doi: 10.1099/00207713-46-4-926

2.- Benedetti P, Rassu M, Pavan G, Sefton A, Pellizzer G. Septic shock, pneumonia, and soft tissue infection due to Myroides odoratimimus: report of a case and review of Myroides infections. Infection 2011; 39: 161-5. doi: 10.1007 / s15010-0100077-1.

3.- LaVergne S, Gaufin T, Richman D. Myroides injenensis bacteremia and severe cellulitis. Open Forum Infect Dis 2019; 6: ofz282. doi: 10.1093/ofid/ofz282.

4.- Licker M, Sorescu T, Rus M, Cirlea N, Horhat F, Jurescu C, et al. Extensively drug-resistant Myroides odoratimimus - a case series of urinary tract infections in immunocompromised patients. Infect Drug Resist 2018; 11: 743-9. doi: 10.2147 / IDR.S161069.

5.- Douce R W, Zurita J, Sánchez O, Cardenas

Aldaz P. Investigation of an outbreak of central venous catheter-associated bloodstream infection due to contaminated water. Infect Control Hosp Epidemiol 2008; 29: 364-6. doi: 10.1086 / 533543.

6.- Ferrer C, Jakob E, Pastorino G, Juncos L I. Right-sided bacterial endocarditis due to Flavobacteriun odoratum in a patient on chronic haemodialysis. Am J Nephrol 1995; 15 : 82-4. doi: 10.1159 / 000168806.

7.- Lorenzin G, Piccinelli G, Carlassara L, Scolari F, Caccuri F, Caruso A, et al. A. Myroides odoratimimus urinary tract infection in an immunocompromised patient: an emerging multidrug-resistant micro-organism. Antimicrob Resist Infect Control 2018; 7: 96. doi: 10.1186/ s13756-018-0391-4.

8.- Maraki S, Sarchianaki E, Barbagadakis S. Myroides odoratimimus soft tissue infection in an immunocompetent child following a pig bite: case report and literature review. Braz J Infect Dis 2012; 16: 390-2. doi: 10.1016/j. bjid.2012.06.004..

9.- Beharrysingh R. Myroides bacteremia: A case report and concise review. IDCases. 2017; 8: 34-6. doi: 10.1016/j.idcr.2017.02.012.

10.- Pérez-Lazo G, Morales-Moreno A, SotoFebres F, Jove-Químper H, Morales-Castillo L, Palomares-Reyes C, et al. First report of Myroides phaeus bacteraemia identified by polymerase chain reaction and genetic sequencing. IDCases. 2020; 19: e00695. doi:10.1016/j.idcr.2020.e00695.

11.- Dharne M S, Gupta A K, Rangrez A Y, Ghate H V, Patole M S, Shouche Y S. Antibacterial activities of multi drug resistant Myroides odoratimimus bacteria isolated from adult flesh flies (Diptera: sarcophagidae) are independent of metallo beta-lactamase gene. Braz J Microbiol 2008; 39: 397-404. doi: 10.1590/ S1517-83822008000200035.

12.- Bachmeyer C, Entressengle H, Khosrotehrani K, Goldman G, Delisle F, Arlet G, et al. Cellulitis due to Myroides odoratimimus in a patient with alcoholic cirrhosis. Clin Exp Dermatol 2008; 33: 97-8. doi: 10.1111/j.13652230.2007.02590.x. 